\title{
FOLIATIONS WITH A RADIAL KUPKA SET ON PROJECTIVE SPACES
}

\author{
OMEGAR CALVO-ANDRADE
}

\begin{abstract}
We consider the set $K(n, c, x d y-y d x)$ of codimension one holomorphic foliations on $\mathbb{P}^{n}, n \geq 3$, with Chern class $c$, and with a compact, connected Kupka set of radial transversal type. We will prove that foliations in this set, have a rational first integral and define an irreducible component of the space of foliations.
\end{abstract}

0.1. Introduction. Let $n \geq 3$ and $c \geq 2$ be natural numbers. Consider a differential 1 -form in $\mathbb{C}^{n+1}$

$$
\omega=a_{0} d z_{0}+\cdots+a_{n} d z_{n}, \in
$$

where $a_{j}$ are homogeneous polynomials of degree $c-1$ in variables $z_{0}, \ldots, z_{n}$ with complex coefficients. Let us assume that

$$
z_{0} a_{0}+\cdots+z_{n} a_{n}=0
$$

so that $\omega$ descends to the complex projective space $\mathbb{P}^{n}$ and defines a global section of the twisted sheaf of 1 -forms $\Omega_{\mathbb{P} n}^{1}(c)$.

For a $\mathbb{K}$-vector space $V$, we denote $\mathbb{P} V=V-\{\mathbf{0}\} / \mathbb{K}^{*}$ the projective space of linear subspaces of $V$ and $\pi: V-\{\mathbf{0}\} \rightarrow \mathbb{P} V$ the quotient map.

Consider the projective space $\mathbb{P} H^{0}\left(\mathbb{P}^{n}, \Omega^{1}(c)\right)$ and the subset

$$
\mathcal{F}(n, c):=\pi\left(\left\{\omega \in H^{0}\left(\mathbb{P}^{n}, \Omega_{\mathbb{P} n}^{1}(c)\right)-\mathbf{0} \mid \omega \wedge d \omega=0\right\}\right)
$$

parameterizing 1-forms $\omega$ such that they satisfy the Frobenius integrability condition. This is the space of Chern class $c$ foliations of codimension one on $\mathbb{P}^{n}$. It is an algebraic subset defined by quadratic equations, and has several irreducible components. For instance, let us recall (see [CL96, GML91]) the following families of irreducible components:

The rational components $R_{n}(a, b) \subset \mathcal{F}(n, c)$ consisting of integrable 1forms of the type

$$
\omega=p g d f-q f d g \in H^{0}\left(\mathbb{P}^{n}, \Omega^{1}(c)\right)
$$

where $c=a+b$ is a partition with $a, b$ natural numbers, $p, q$ are the unique coprime numbers such that $p a=q b$ and $f, g$ are homogeneous polynomials of respective degree $a, b$.

1991 Mathematics Subject Classification. 58A17, 32G99.

Key words and phrases. Kupka set, ample vector bundle.

MTM2004-07978, Spain. 
The leaves of the foliation $\omega=p g d f-q f d g \in R_{n}(a, b)$, are the fibers of the rational map $\varphi=f^{p} / g^{q}$, and we say that $\varphi$ is a rational first integral of the foliation $\omega$.

The singular set $S(\omega)=\left\{p \in \mathbb{P}^{n} \mid \omega(p)=0\right\}$, of a foliation $\omega \in \mathcal{F}(n, c)$, is the variety of zeros of the section $\omega$. We set $S_{k}(\omega)$, the union of the irreducible components of $S(\omega)$ of dimension $k$. It is always possible to suppose that $\operatorname{codim}(S(\omega)) \geq 2$. Namely, if $S_{n-1}(\omega)$ is not empty (i.e. if $\omega$ vanishes in codimension one). Then there exists a homogeneous polynomial $F$ of maximal degree $0<e<c-1$ that divides $\omega$. We denote

$$
\bar{\omega}=\frac{\omega}{F} \in H^{0}\left(\mathbb{P}^{n}, \Omega_{\mathbb{P}^{n}}^{1}(c-e)\right) .
$$

It is clear that $\bar{\omega}$ is well defined up to a multiplicative constant and it does not vanish in codimension one.

In this paper, the Rational Components $R_{n}(c / 2, c / 2)$ will be characterized by its singular set, our main hypothesis, is that $S_{n-2}(\omega)$ is compact, connected and it is of Kupka type. The Kupka set is defined by

$$
K(\omega)=\left\{p \in \mathbb{P}^{n} \mid \omega(p)=0, \quad d \omega(p) \neq 0\right\} \subset S(\omega) .
$$

The main properties of Kupka sets, are the following

(a): $K(\omega)$ is smooth of codimension two.

(b): It is stable under deformations of the foliation.

(c): $K(\omega)$ has local product structure.

We are going to consider the subset of foliations

$$
K(n, c)=\left\{\omega \in \mathcal{F}(n, c) \mid S_{n-2}=K(\omega) \text { is compact and connected }\right\}
$$

The stability under deformations of the Kupka set, implies that $K(n, c)$ is an open subset of $\mathcal{F}(n, c)$, its closure, is a union of irreducible components. On the other hand, a fundamental fact about a foliation $\omega \in K(n, c)$, is that $\omega$ has a rational first integral and belongs to some irreducible rational component $R_{n}(a, b)$; if and only if, its Kupka set $K(\omega)$, is a complete intersection [CL92]. Furthermore, it is conjectured that $K(\omega)$ is always a complete intersection CL92. There are already several partial positive answers to this conjecture. In fact: in [C99, Theorem 3.5] it is proven that for foliations $\omega \in K(n, c)$ which are not of radial type, there always is a rational first integral, and in [B95, B99], we find the hypothesis $n \geq 6$. The conjecture remains open for the radial transversal type in small dimensions.

In this note we are going to prove the following result:

Theorem 0.1. The set $K(n, c, x d y-y d x) \subset K(n, c)$ of foliations of radial type is not empty if and only if $c$ is even, moreover there are homogeneous polynomials $f, g$ of degree $c / 2$ such that $K(\omega)$ is the scheme theoretically complete intersection $K(\omega)=\{f=g=0\}$ and

$$
\omega=f d g-g d f \text {. }
$$


We observe that for any even natural number $c$, the generic element of the irreducible component $R_{n}(c / 2, c / 2) \subset \mathcal{F}(n, c)$, belongs to the set $K(n, c)$. Theorem (0.1) states that the closure of $K(n, c, x d y-y d x)$ is precisely the set $R_{n}(c / 2, c / 2)$, therefore, it is an irreducible component of the space of foliations $\mathcal{F}(n, c)$.

As a consequence of Theorem (0.1), the results in GML91 and in C99, Theorem 3.5], we obtain as a Corollary, a complete classification of the family of foliations in $K(n, c)$.

Corollary 0.2. Let $\omega \in K(n, c)$ be a foliation, then $K(\omega)$ is a complete intersection and moreover

(1) $\omega$ has a meromorphic first integral.

(2) The sets $K\left(n, c, \eta_{p q}\right)=R_{n}\left(\frac{c p}{p+q}, \frac{c q}{p+q}\right)$, so that they are irreducible components of the set $\mathcal{F}(n, c)$.

(3) The generic element of $K\left(n, c, \eta_{p q}\right)$ is structurally stable.

The proof of the Theorem (0.1) uses the ideas introduced in CS94 and CMP06, theory of foliations by curves in the projective plane $\mathbb{P}^{2}$ and intersection theory, and it will be a consequence of several lemmas.

0.2. Properties of the Kupka Set. From [M77, GML91 and the references therein, it is known that the Kupka set is a smooth submanifold of codimension two and has the local product structure: For any connected component $K_{0} \subset K(\omega)$ of the Kupka set, there are an open covering $\mathcal{U}=\left\{U_{i}\right\}$ of $K_{0}$, a collection of submersions $\psi_{i}: U_{i} \rightarrow \mathbb{C}^{2}$ such that $K_{0} \cap U_{i}=\psi_{i}^{-1}(0)$, as well a holomorphic 1 -form $\eta=a(x, y) d x-b(x, y) d y$ with a unique singularity at $(0,0)$, such that the foliation $\left.\omega\right|_{U_{i}}$ is represented by $\psi_{i}^{*} \eta$. The one form $\eta$ is called the transversal type at the component $K_{0}$, and it is unique up to both biholomorphism and multiplication by a never vanishing holomorphic function.

It is also proved in GML91 that, when the connected component $K_{0}$ is compact, and its normal bundle $N_{K_{0}}$ has non zero first Chern class, the transversal type may be represented by a 1 -form with Taylor expansion $\eta=$ $\eta_{p q}+\cdots$, where $\eta_{p q}=p x d y-q y d x$ with $p, q$ coprime integer or $p=q=1$. Furthermore, when the linear part $\eta_{p q}$ belongs to the Poincaré domain, the transversal type is analytically linearizable. The radial type corresponds to $\eta_{11}=x d y-y d x$. We denote in this case $R=K(\omega)$.

As we will see in the next section, the normal bundle of a compact connected component of the Kupka set, of a foliation of the projective space $\mathbb{P}^{n}, n \geq 3$, always has a non zero first Chern class, and its transversal type has linear part in the Poincaré domain.

0.3. Geometric Properties of Kupka Components. The local product property, implies that for any foliation $\omega \in K(n, c)$, it's Kupka set $K=K(\omega)$ 
is subcanonically embedded, CS94, Theorem 3.4]. In fact, we have

$$
\wedge^{2} N_{K} \simeq \mathcal{O}_{K}(c), \text { and } \Omega_{K}^{n-2} \simeq \mathcal{O}_{K}(c-n-1),
$$

where $\Omega_{K}^{n-2}$ denotes the canonical bundle of the Kupka set $K$.

This property has two consequences:

(a): The first Chern class $c_{1}\left(N_{K}\right) \in H^{2}(K, \mathbb{Z})$ does not vanish. In fact, since $\wedge^{2} N_{\mathbb{K}} \simeq \mathcal{O}_{K}(c)$ we have that $c_{1}\left(N_{K}\right)=\jmath^{*}(c \cdot \mathbf{h})$, where $\jmath: K \hookrightarrow \mathbb{P}^{n}$ denotes the inclusion map, and $\mathbf{h}$ denotes the class of a hyperplane, i.e. generator of the cohomology ring

$$
H^{*}\left(\mathbb{P}^{n}, \mathbb{Z}\right) \simeq \frac{\mathbb{Z}[\mathbf{h}]}{\mathbf{h}^{n+1}}
$$

(b): By a Serre construction, the normal bundle $N_{K}$ of $K$ in $\mathbb{P}^{n}$, extends to a rank two holomorphic vector bundle $V$ of $\mathbb{P}^{n}$, having a holomorphic section $\sigma$, vanishing on $K(\omega)$, and defining the exact sequence

$$
0 \longrightarrow \mathcal{O} \stackrel{\cdot \sigma}{\longrightarrow} V \longrightarrow \mathcal{J}_{K}(c) \rightarrow 0
$$

where $\mathcal{J}_{K}$ denotes the sheaf of ideals of the Kupka set $K$.

Since $K$ is smooth, the total Chern class of the bundle $V$ is

$$
c(V)=1+c \cdot \mathbf{h}+\operatorname{deg}(K(\omega)) \cdot \mathbf{h}^{2} \in \frac{\mathbb{Z}[\mathbf{h}]}{\mathbf{h}^{n+1}} \simeq H^{*}\left(\mathbb{P}^{n}, \mathbb{Z}\right) .
$$

The Kupka set $K$ is a complete intersection if and only if, the holomorphic vector bundle $V$ splits holomorphically into a direct sum of holomorphic line bundles.

In what follows, we are going to looking for a condition for the splitting of the vector bundle $V$, associated to the Kupka set.

Observe that $c_{1}(V)>0$ and $c_{2}(V)>0$, and $c_{1}\left(N_{K}\right) \neq 0$, then the linear part of the transversal type $\eta$ is $\eta_{p q}$ for some coprime integers $p, q$. Now, the Baum-Bott residues formula BB72, implies that

$$
\operatorname{deg}(K(\omega))=\frac{c p}{p+q} \frac{c q}{p+q}
$$

and the linear part $\eta_{p q}$ belongs to the Poincaré domain, consequently on a neighborhood of each point of the Kupka set, the foliation looks like the base points of a rational map. Moreover, we get the decomposition

$$
K(n, c)=\bigcup_{(p, q) \in \mathcal{K}(c)} K\left(n, c, \eta_{p q}\right),
$$

where $K\left(n, c, \eta_{p q}\right)$ denotes the set of foliations $\omega \in K(n, c)$ with transversal type $\eta_{p q}=p x d y-q y d x$, and we set

$$
\mathcal{K}(c)=\left\{(p, q) \in \mathbb{N} \times \mathbb{N} \mid\left(\frac{p c}{p+q}, \frac{q c}{p+q}\right) \in \mathbb{N} \times \mathbb{N}\right\}
$$


The total Chern class of the vector bundle $V$ may be written

$$
c(V)=\left(1+\frac{c p \cdot \mathbf{h}}{p+q}\right)\left(1+\frac{q c \cdot \mathbf{h}}{p+q}\right)
$$

In particular, if the transversal type is the radial foliation $x d y-y d x$, we have the following result

Lemma 0.3. Let $\omega \in K(n, c, x d y-y d x)$ then

(a): The Chern class of the foliation is even.

(b): The total Chern class of the associated vector bundle $V$ is

$$
c(V)=\left(1+\frac{c \cdot \mathbf{h}}{2}\right)^{2}
$$

(c): The degree of the Kupka set is

$$
\operatorname{deg}(K)=\frac{c^{2}}{4}
$$

Proof. Observe that equations (3) and (4) imply

$$
c(V)=\left(1+\frac{c \cdot \mathbf{h}}{2}\right)^{2} \text { and } \operatorname{deg}(K(\omega))=\frac{c^{2}}{4}=\left(\frac{c}{2}\right)^{2},
$$

therefore, c must be even.

0.4. Holomorphic Vector Bundles on the Projective Space. Let us recall some notions of holomorphic vector bundles on the projective space. Our main reference in this section is OSS78.

For a rank two holomorphic vector bundle $V$, which has even first Chern class $c=c_{1}(V)$, put

$$
V_{\text {norm }}:=V\left(-\frac{c}{2}\right)
$$

and it is normalized in order to have $c_{1}\left(V_{\text {norm }}\right)=0$.

We say that $V$ is stable if $H^{0}\left(\mathbb{P}^{n}, V_{\text {norm }}\right)=0$ and $V$ is semistable if $H^{0}\left(\mathbb{P}^{n}, V_{\text {norm }}\right) \neq 0$ and $H^{0}\left(\mathbb{P}^{n}, V_{\text {norm }}(-1)\right)=0$.

Recall that the vector bundle $V$ associated to a Kupka component never is stable [CS94, Corollary 4.3]. For a foliation $\omega \in K(n, c, x d y-y d x)$ with Kupka set $R$, the total Chern class is compatible with the semiestability. A Particular case of the next result was proved and used in [CMP06].

Lemma 0.4. Let $\omega \in K(n, c, x d y-y d x)$ be a foliation of the projective space $\mathbb{P}^{n}$ with Kupka component $R=K(\omega)$ and associated vector bundle $V$. If the holomorphic vector bundle $V$ is semistable and not stable, then $R$ is a complete intersection.

Proof. Let $\tau$ be a non trivial holomorphic section of the bundle $V_{\text {norm }}$, and let $Z$ be the its scheme of zeros. If $\operatorname{codim}(Z)=1$, and $Z_{n-1}$ denotes the union of irreducible components of dimension $n-1$ (i.e. codimension one), then there is a homogeneous polynomial $F$ of maximal degree $0<e$ such 
that $Z_{n-1}=\{F=0\}$. It follows that $(\tau / F) \in H^{0}\left(\mathbb{P}^{n}, V_{\text {norm }}(-e)\right)$ is a non zero section, contradicting the semistability of $V$.

If $\operatorname{codim}(Z)=2$, we have the exact sequence

$$
0 \longrightarrow \mathcal{O} \stackrel{\cdot \tau}{\longrightarrow} V_{\text {norm }} \longrightarrow \mathcal{J}_{Z} \longrightarrow 0
$$

We have that $\operatorname{deg}(Z)=c_{2}\left(V_{\text {norm }}\right)=0$, then $Z$ is empty and $V_{\text {norm }}$ is obtained as an extension of the trivial line bundle by the trivial line bundle, hence $V_{\text {norm }} \simeq \mathcal{O} \oplus \mathcal{O}$. Then

$$
V \simeq V_{\text {norm }}\left(\frac{c}{2}\right) \simeq \mathcal{O}\left(\frac{c}{2}\right) \oplus \mathcal{O}\left(\frac{c}{2}\right)
$$

Therefore, the question of when a foliation $\omega \in K(n, c, x d y-y d x)$ has a meromorphic first integral, is reduced to deciding when the rank two holomorphic vector bundle $V$ associated with the Kupka set $R=K(\omega)$ is semistable.

We are going to use the following geometric criteria, in order to prove the semistability of a rank two vector bundle on $\mathbb{P}^{n}$ which belongs to a locally complete intersection. The proof of the following lemma may be found in OSS78, page 186].

Lemma 0.5. Let $V$ be a rank two holomorphic vector bundle which belongs to the 2 codimensional locally complete intersection $Y \subset \mathbb{P}^{n}, \quad n \geq 3$ and with $\wedge^{2} N_{Y}=\mathcal{O}_{Y}(c)$. If $c$ is even, then $V$ is semistable if and only if $c \geq 0$ and $Y$ lies in no hypersurface of degree $d<c / 2$.

By [CS94, Corollary 4.3], the Kupka of a foliation $\omega \in K(n, c, x d y-y d x)$ is contained on an hypersurface of degree $d \leq c / 2$. A combination of the lemmas (0.5) and (0.4) implies that $\omega$ in $K(n, c, x d y-y d x)$, has a meromorphic first integral if it's Kupka set $R=K(\omega)$ no lies in no hypersurface of degree $d<c / 2$. We are going to estimate the degree of an hypersurface $H$ such that $R \subset H$.

0.5. The Normal Bundle of $R$. Given a foliation $\omega \in K(n, c, x d y-y d x)$, with Kupka set $R$, we will see that $R$ can not be contained in the smooth points of an hypersurface $H$ of degree $d<c / 2$. In order to prove this, we remark the following properties of the normal bundle $N_{R}$ of $R$ in $\mathbb{P}^{n}$.

The first remark is the following: The normal bundle $N_{R}$ of $R$ in $\mathbb{P}^{n}$, is projectively flat, i.e. the projective bundle $\mathbb{P}\left(N_{R}\right)$ is flat. This property is a consequence of the fact that, the strict transformation of the foliation obtained by the blowing up along $R$ of a foliation $\omega \in K(n, c, x d y-y d x)$, is transversal to the exceptional divisor $E=\mathbb{P}\left(N_{R}\right)$. The intersection of this foliation with $E$, provides the flat structure on the projective bundle $\mathbb{P}\left(N_{K}\right)$.

On the other hand, for any $\alpha \in \mathbb{Z}$, the holomorphic vector bundle $N_{R}(\alpha)$ is also projectively flat. Moreover, for any $0<d<c / 2$, the vector bundle $N_{R}(-d)$ is projectively flat and has positive first Chern class. 
The curvature matrix $\widetilde{\Theta}=\Theta\left(N_{R}(-d)\right)$ of the twisted normal bundle $N_{R}(-d)$ is of the type

$$
\widetilde{\Theta}=\Theta \cdot \mathbf{I},
$$

where $\mathbf{I} \in \operatorname{End}\left(N_{R}\right)$ is the identity endomorphism, and $\Theta$ is a positive 2form on $R\left[\right.$ K87, page 7]. Therefore, the twisted normal bundle $N_{R}(-d)$ is Griffiths positive G69, and hence, it is ample.

We are going to use the following result, which implies the semistability of the vector bundle $V$, associated to the Kupka set $R$. The proof may be found in the book [FOV99, page 133].

Lemma 0.6. Let $X \subset \mathbb{P}^{n}$ be a smooth subvariety such that the twisted normal bundle $N_{X}(-d)$ is ample. Let $H \subset \mathbb{P}^{n}$ by a hypersurface of degree d. Then the set of tangencies

$$
\Gamma=\left\{x \in X \cap H_{\text {reg }} \mid T_{x} X \subset T_{x} H\right\}
$$

is 0 -dimensional

0.6. Foliations and Curves in Surfaces. The main reference through this section, is the book Br00.

Recall that a holomorphic foliation $\mathcal{F}$ by curves on a surface $M$, may be represented as either

- By a bundle map $\mathbf{X}: T_{\mathcal{F}} \rightarrow T M$.

- By a holomorphic section $\omega$ of the twisted sheaf of one forms $\Omega_{M}^{1}\left(N_{\mathcal{F}}\right)$. where $T_{\mathcal{F}}$ and $N_{\mathcal{F}}$ are holomorphic line bundles over $M$, called respectively the tangent and the normal bundle of the foliation. These line bundles are related by the formula

$$
\Omega_{M}^{2}=T_{\mathcal{F}}^{*} \otimes N_{\mathcal{F}}^{*} .
$$

The above formula, gives for a foliation $\mathcal{F}$ on the projective plane $\mathbb{P}^{2}$, represented by a section $\omega$ of the twisted sheaf $\Omega_{\mathbb{P}^{2}}^{1}(c)$, has a normal bundle $N_{\mathcal{F}}=\mathcal{O}_{\mathbb{P}^{2}}(c)$ and its tangent bundle $T_{\mathcal{F}}=\mathcal{O}_{\mathbb{P}^{2}}(3-c)$.

Consider a foliation $\omega \in \mathcal{F}(2, c)$, and assume that there is an irreducible algebraic curve $C \subset \mathbb{P}^{2}$ of degree $d$. We are going find some relations between the degree of the curve $C$, with the Chern class of the foliation, depending of course, on the behavior of the curve with respect the foliation. We consider two cases:

(1) Non invariant curves.

(2) Invariant curves.

(1) Following Br00, page 23], given a foliation $\mathcal{F}$ by curves on a surface $M$, and a non $\mathcal{F}$-invariant curve $C$, for each $p \in C$, we define the index $\tan (\mathcal{F}, C, p)$, representing the tangency order of the foliation $\mathcal{F}$ with the curve $C$ at $p$ as follows: let $\{f=0\}$ be a local equation of $C$ around $p$, 
and let $\mathbf{X}$ a vector field with isolated singularities and defining the foliation around $p$. We set

$$
\operatorname{tang}(\mathcal{F}, C, p)=\operatorname{dim}_{\mathbb{C}} \frac{\mathcal{O}_{p}}{\langle\mathbf{X}(f), f\rangle},
$$

remark that $\mathbf{X}(f)$ does not vanishes identically on $C$ because it is not $\mathcal{F}$ invariant, and so, the ideal $\langle\mathbf{X}(f), f\rangle$, has finite codimension on $\mathcal{O}_{p}$ and $\operatorname{tang}(\mathcal{F}, C, p)$ is a finite non negative integer, moreover it is not zero at points where $C$ is not transverse to $\mathcal{F}$, of course, the singularities of the curve, and the singularities of the foliations at the curve, are tangency points of the curve $C$ with the foliation $\mathcal{F}$.

For instance, let $\mathbf{X}=x \partial / \partial x+y \partial / \partial y$ and $C=\{f=0\}$ a curve through $0 \in \mathbb{C}^{2}$. Assume that the curve is singular at $0 \in \mathbb{C}^{2}$, then it may be written as $f=f_{\nu}+f_{\nu+1}+\cdots, \quad \nu \geq 2$. In this case, the index $\operatorname{tang}(\mathcal{F}, C, 0) \geq 3$, since

$$
\langle\mathbf{X}(f), f\rangle=\left\langle\nu f_{\nu}+\cdots, f_{\nu}+\cdots\right\rangle=\langle g, f\rangle,
$$

and $g=\nu \cdot f_{\nu+1}+\cdots$.

Now, we define

$$
\operatorname{Tang}(\mathcal{F}, C)=\sum_{p \in C} \operatorname{tang}(\mathcal{F}, C, p)
$$

(2) If the curve $C$ is invariant by the foliation $\omega$; we introduce the following index $Z(\mathcal{F}, C, p)$ for any $p \in C$ as follows (see [Br00, page 24]). Let $f=0$ be any local equation of the curve $C$ at $p$, and let $\theta$ be a generating 1 -form of the foliation $\mathcal{F}$ at $p$. Because $C$ is invariant, we have

$$
g \cdot \theta=h d f+f \eta, \quad g, h \in \mathcal{O}_{p} \quad \eta \in \Omega_{p}^{1},
$$

moreover $(f, h)=1$ in $\mathcal{O}_{p}$, therefore $h, g$ does not vanish identically on any branch of $C$. We can define

$$
Z(\mathcal{F}, C, p)=\text { vanishing order of }\left(\left.\frac{f}{g}\right|_{C}\right) \text { at } p \text {. }
$$

and assuming that $C$ is compact, we define

$$
Z(\mathcal{F}, C)=\sum_{p \in C} Z(\mathcal{F}, C, p) .
$$

This idex could be non positive for dicritical singularities, for example, let $\omega=x d y-y d x$ be a 1 -form generating a foliation on $\mathbb{C}^{2}$, let $C=\{f=0\}$ be an invariant curve by the foliation around $p=0$. If $C$ is smooth (we can take $f=x)$, then $Z(\mathcal{F}, C, 0)=1$, but it is non positive in if the curve $C$ is singular.

The result that we need is the following:

Proposition 0.7. Let $\omega \in \mathcal{F}(2, c)$ be a foliation on the projective plane $\mathbb{P}^{2}$. Let $C$ be an irreducible algebraic curve of degree $d$ in $\mathbb{P}^{2}$. Then 
- If the curve $C$ is invariant by the foliation $\omega$ we have

$$
Z(\mathcal{F}, C)=c \cdot d-d^{2}
$$

- If the curve $C$ is not invariant by the foliation $\omega$.

$$
\operatorname{Tang}(\mathcal{F}, C)=d^{2}+(c-3) \cdot d
$$

We can now complete the proof of the Theorem.

Proof of theorem (0.1).

Let $\omega \in K(n, c, x d y-y d x)$, by lemma (0.4), it is sufficient to prove that the associated vector bundle $V$ is semistable.

If the vector bundle $V$ is not semistable, since $R$ is irreducible, then there exists an irreducible hypersurface $H$ of degree $d<c / 2$ such that $R \subset H$. By the lemma (0.6), $R$ is not contained in the smooth points of the hypersurface $H$.

Take a two plane $\jmath: \mathbb{P}^{2} \hookrightarrow \mathbb{P}^{n}$ in generic position with respect the foliation and the hypersurface $H$. Consider the curve $C=\jmath^{-1}(H)$, and the foliation $\jmath^{*} \omega \in \mathcal{F}(2, c)$. This foliation has $\left\{p_{j}\right\}_{j=1, \ldots c^{2} / 4}=J^{-1}(R)$ radial singularities $(\jmath \pitchfork R)$, and the other singularities are Morse type (i.e. there is a local coordinate system $(x, y)$ such that $\left.\jmath^{*} \omega=d(x y)\right)$. Moreover, the radial singularities are contained in the curve $C$.

If the hypersurface $H$ is not invariant, the same holds for the curve $C$, moreover, because $C$ is singular at the points $\left\{p_{j}\right\}$ we have seen that

$$
\operatorname{tang}\left(\jmath^{*} \omega, C, p_{j}\right) \geq 3 \text { for any } p_{j} \in \jmath^{-1}(R) .
$$

and then, by equation (7) of proposition (0.7) we have that $d>c / 2$.

If the hypersurface $H$ is invariant, then $Z\left(\jmath^{*} \omega, C, p_{j}\right)$ is non positive, and then, by formula (6) of proposition (0.7), its degree $d \geq c>c / 2$, therefore we have a contradiction, therefore, the vector bundle $V$ is semistable.

By Lemma (0.5), the holomorphic vector bundle $V$ associated to $R$ is semistable and by Lemma (0.4) it is isomorphic to

$$
V \simeq \mathcal{O}(c / 2) \oplus \mathcal{O}(c / 2)
$$

Therefore, after the isomorphism between $V$ and $\mathcal{O}(c / 2) \oplus \mathcal{O}(c / 2)$, the section $\sigma=(f, g)$, for some $f, g \in H^{0}\left(\mathbb{P}^{n}, \mathcal{O}_{\mathbb{P}^{n}}(c / 2)\right)$. Hence, the Kupka set $R$ is a complete intersection and by Cerveau-Lins theorem CL92, the foliation has a rational first integral, and it is defined by the 1-form

$$
\omega=f d g-g d f .
$$

This finishes the proof of the Theorem 0.1

Remark 0.8. Let $M$ be an algebraic manifold with $\operatorname{dim}_{\mathbb{C}} M \geq 3$. Consider the set $K(M, L, x d y-y d x)$ of those foliations $M$ with normal bundle $L$, (i.e. represented by a section $\omega$ of the sheaf $\Omega_{M}^{1}(L)$ ), and having a compact and connected radial Kupka component. 
If the line bundle $L$ is positive, there exists the pair a rank two holomorphic vector bundle $V \rightarrow M$ with a section $\sigma$ inducing the exact sequence

$$
0 \longrightarrow \mathcal{O} \stackrel{\cdot \sigma}{\longrightarrow} V \longrightarrow \mathcal{J}_{K}(L) \rightarrow 0 .
$$

The vector bundle $V$ has total Chern class

$$
c(V)=\left(1+\frac{c_{1}(L)}{2}\right)^{2} \in H^{*}(M, \mathbb{Z})
$$

but in general, the vector bundle $V$, is not semistable, as was proved for the projective space.

In the general case, we have the following conjecture.

Conjecture 0.9. Let $\omega \in K(M, L, x d y-y d x)$ where $L$ is a very ample line bundle, then the associated vector bundle $V$ is projectively flat.

This means that any foliation $\omega \in K(M, L, x d y-y d x)$, has a projectively transversal structure. Moreover, if we consider the section $[\sigma]$, as a meromorphic section of the projective bundle $\mathbb{P}(V)$, it has the base locus at $R$, and it defines the developing map of the transversal structure of the foliation.

\section{REFERENCES}

[BB72] Baum, P., Bott, R. Singularities of Holomorphic Foliations. Journal on Diferential Geometry 7, 279-342, 1972.

[B95] Ballico, E. A splitting theorem for the Kupka component of a foliation of $\mathbb{C P}^{n}, n \geq 6$. Addendum to a paper by Calvo-Andrade and Soares. Ann. Inst. Fourier 45, 1119-1121, 1995.

[B99] Ballico, E. A splitting theorem for the Kupka component of a foliation of $\mathbb{C P}^{n}, n \geq 6$. Addendum to an addendum to a paper by Calvo-Andrade and Soares. Ann. Inst. Fourier 49, 1423-1425, 1999.

[Br00] Brunella, M. Birrational Geometry of Foliations. First Latin American Congress of Mathematicians (2000), Monographs of IMPA (www.impa.br).

[C99] Calvo, O. Foliations with a Kupka Component on Algebraic Manifolds. Bull. of the Brazilian Math. Soc. 30 No. 2, 183-197, 1999.

[CMP06] Calvo O., Mendes L.G., Pan, I. Foliations with radial Kupka set and pencils of Calabi-Yau hypersurfaces. Compositio Math. 142, 1587-1593, 2006.

[CS94] Calvo, O., Soares, M. Chern numbers of a Kupka component. Ann. Inst. Fourier 44, 1219-1236, 1994.

[CL92] Cerveau, D., Lins, A. Codimension one Foliations in $\mathbb{C P}^{n}, \quad n \geq 3$, with Kupka components. in Complex Analytic Methods in Dynamical Systems. C. Camacho, A. Lins, R. Mossou, P. Sad. Eds. Astérisque 222, 93-133, 1994.

[CL96] Cerveau, D., Lins, A. Irreducible components of thespace of holomorphic foliations of degree two in $\mathbb{C P}(n)$. Annals of Math. 143, 577-612, 1996.

[FOV99] Flenner, H., O'Carrol L., Vogel, W.: Joins and Intersections. Springer Monographs in Math, 1999.

[GML91] Gómez-Mont X., Lins A. Structural Stability of foliations with a meromorphic first integral Topology 30, 315-334, 1991.

[G69] Griffiths Ph. Hermitian Differential Geometry, Chern Classe and Positive Vector Bundles in Global Analysis, papers in honor of K. Kodaira. Tokyo, Princenton: University of Tokyo Press, Princenton University Press 185-251, 1969. 
[GH78] Griffiths Ph., Harris J. Principles of Algebraic Geometry. Pure \& Appl. Math. Wiley Interscience, 1978.

[K87] Kobayashi S. Differential Geometry of Complex Vector Bundles. Kano Memorial Lectures 5, Princeton University Press, 1987.

[M77] Medeiros A. Structural stability of integrable differential forms. in Geormetry and Topology J. Palis, M. do Carmo Eds. Springer LNM 597, 395-428, 1977.

[OSS78] Okonek Ch., Schneider M., Spindler H. Vector Bundles on Complex Projective spaces. Progress in Math. 3, Birkhauser 1978.

Cimat: Ap. Postal 402, Guanajuato, 36000, Gto. México

E-mail address: omegar@cimat.mx 